Talk presented at "Lattice 2003", July 15-19, 2003, Tsukuba, Japan

KEK-TH-915

RBRC-334

\title{
Nucleon axial charge and structure functions with domain wall fermions
}

\author{
Shigemi Ohta ${ }^{\text {ab }}$ and Kostas Orginos ${ }^{\mathrm{b}}$ for the RIKEN-BNL-Columbia-KEK Collaboration* \\ anstitute of Particle and Nuclear Studies, KEK, Tsukuba, Ibaraki 305-0801, Japan \\ ${ }^{b}$ RIKEN BNL Research Center, Brookhaven National Laboratory, Upton, NY 11973, USA
}

We report the current status of RBCK calculations on nucleon structure with both quenched and unquenched lattice QCD. The combination of domain wall fermions and DBW2 gauge action works well for isovector vector and axial charges, and moments of structure functions $\langle x\rangle_{q}, d_{1}$, and $\langle 1\rangle_{\delta q}$.

Domain wall fermions (DWF) 1] have been successfully used by the RBC Collaboration for several applications [2. In the baryon sector we had reported that DWF with Wilson gauge action in the quenched approximation can reproduce the first negative-parity excited state of nucleon 3] which defied theoretical attempts for a long time. Here we report the current status of our calculations on the nucleon electroweak matrix elements, namely the isovector vector and axial charges and some moments of the structure functions, in both quenched and full-QCD, using DWF and the DBW2 gauge action [4.

Four form factors appear in neutron $\beta$ decay: the vector and induced tensor form factors from the vector current, and the axial-vector and induced pseudo-scalar form factors from the axialvector current. In the forward limit, the 4momentum transfer should be small because the mass difference of the neutron and proton is only about $1.3 \mathrm{MeV}$. This makes the limit $q^{2} \rightarrow 0$, where the vector and axial-vector form factors dominate, a good approximation. Their values in this limit are called the vector and axial charges of the nucleon: $g_{V}$ and $g_{A}$.

Lattice numerical calculation of these charges is relatively simple: prepare appropriate nucleon source and sink at rest, insert an appropriate current operator in between, and identify a plateau which gives a lattice estimate. There already had been several preceding works using Wilson or improved Wilson fermions [5]. However since

\footnotetext{
*Talk by SO. We thank RIKEN, Brookhaven National Laboratory and the U.S. Department of Energy for providing the facilities essential for the completion of this work.
}

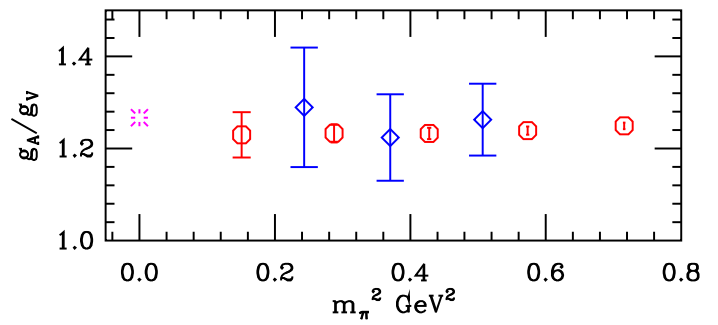

Figure 1. Quark mass dependence of axial to vector charge ratio, $g_{A} / g_{V}$, quenched (circle) and full QCD (diamond). Full-QCD results, albeit with small statistics, are consistent with quenched results: No mass dependence is seen.

these fermions schemes do not respect the chiral symmetry, the lattice renormalizations of the relevant vector and axial currents are not the same. Worse, unwanted lattice artifact may result in unphysical mixing of chirally distinct operators. The combination of DWF and DBW2 preserves chiral symmetry well enough so that we are free of these difficulties: especially the relation $Z_{A}=Z_{V}$ is easily and well maintained, up to $O\left(a^{2}\right)$, or a few $\%$ at $a \approx 0.15 \mathrm{fm}$. And in contrast to those preceding studies, we found a significant finite volume effect between the results calculated on lattices with $(1.2 \mathrm{fm})^{3}$ and $(2.4 \mathrm{fm})^{3}$ volumes [6] about $20 \%$ increase was observed. On the large volume we find $g_{A}=$ $1.212 \pm 0.027$ (stat) \pm 0.024 (norm). The quoted systematic error is the dominant known one, corresponding to current renormalization. This theoretical first principles calculation, which does not yet include isospin breaking effects, yields a 

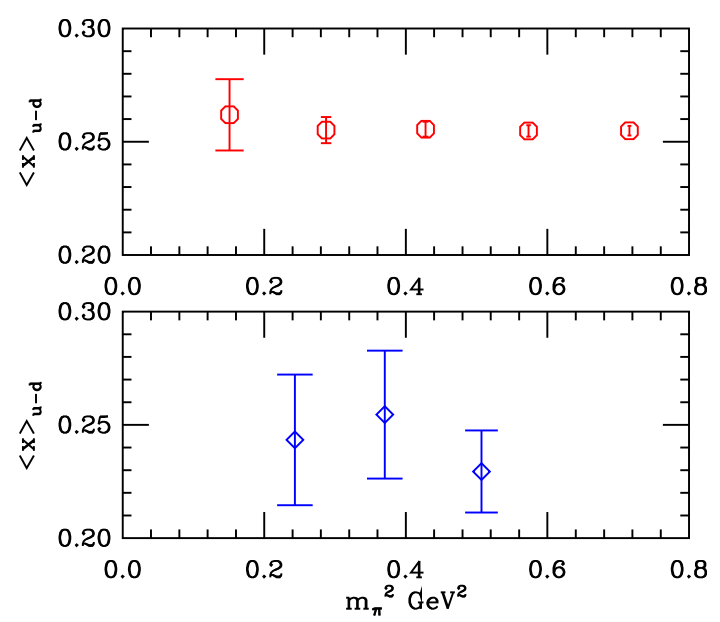

Figure 2. The bare momentum fraction in quenched (circle) and full-QCD (diamond).

value of $g_{A}$ only a little bit below the experimental one, $1.2670 \pm 0.0030$ [7]. The dependence on the quark mass on the larger volume is very mild, while it is steep on the smaller.

First few moments of nucleon structure functions are as easy as these charges, in that they do not require finite momentum states. The structure functions such as $F_{1}\left(x, Q^{2}\right), F_{2}\left(x, Q^{2}\right)$, $g_{1}\left(x, Q^{2}\right)$, and $g_{2}\left(x, Q^{2}\right)$ are measured in lepton deep inelastic scattering, and $h_{1}\left(x, Q^{2}\right)$ in the RHIC Spin experiment. Their moments are factorized in the operator product expansion into Wilson coefficients and non-perturbative quantities such as $\left\langle x^{n}\right\rangle_{q},\left\langle x^{n}\right\rangle_{\Delta q}, d_{n}$ or $\langle 1\rangle_{\delta q}$. The latter are nucleon forward matrix elements of certain local operators, such as $\gamma D, \gamma_{5} \gamma D$, and $\gamma_{5} \sigma D$, and hence are accessible by lattice QCD numerical methods similar to form factor calculations. There are calculations by QCDSF, QCDSF/UKQCD, and LHPC-SESAM collaborations with (improved) Wilson fermions [5]. In Lattice 2002 we presented some preliminary quenched results from the present DWF/DBW2 calculations 8]. The DWF/DBW2 combination indeed works better: good chiral behavior, ease of non-perturbative renormalization, and better approach to the continuum were seen. We present higher statistics quenched results here, and some preliminary full-QCD ones. We still lack non-
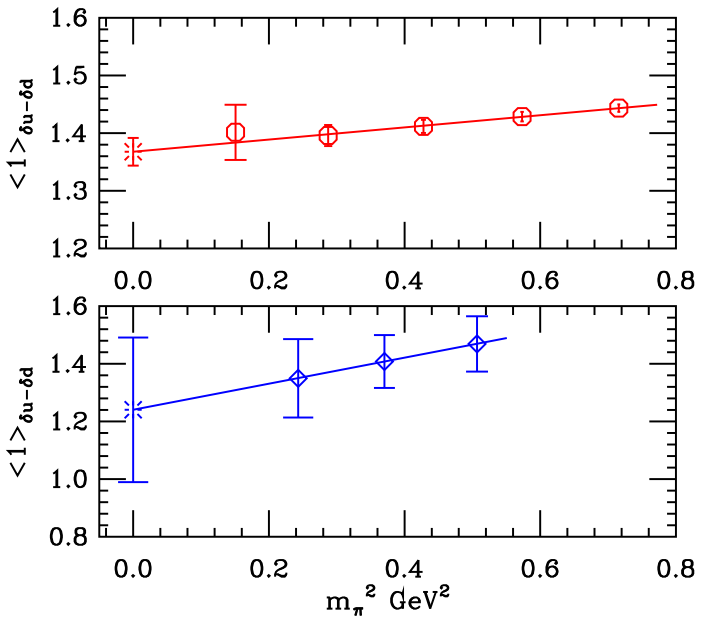

Figure 3. The bare $\langle 1\rangle_{\delta u-\delta d}$ matrix element in the quenched (circle) and full (diamond) QCD.

perturbative renormalization for some observables.

In the on-going full-QCD calculations, a $16^{3} \times$ $32 \times 12$ lattice is used with the DBW2 gauge coupling set at $\beta=0.8$ and the domain-wall height at $M_{5}=1.8$. Dynamical DWF quarks with mass $0.02,0.03$, and 0.04 (about strange mass) 9] in the lattice units are used. The nucleon structure calculations reported here are done only at the dynamical quark mass, and no partially quenched study has been made. About 900-200 conjugategradient iterations are necessary to achieve $10^{-8}$ accuracy for the Dirac equation solver. Hybrid Monte Carlo acceptance of about 70-80\% is observed. About 50 configurations are used at each dynamical quark mass value. The residual mass of $m_{\text {res }}=0.00137, \rho$ meson mass $m_{\rho}=0.43(2)$ which translates into the lattice cutoff estimate of $a^{-1} \sim 1.8 \mathrm{GeV}$, and the nucleon mass $m_{N}=$ $0.56(4)$ or $\sim 1.01(7) \mathrm{GeV}$ are obtained. Quality of plateaus in the three-point functions is similar to the quenched case at similar statistics.

In Fig. 11 we show the ratio of the isovector axial and vector charges as a function of the pion mass squared. The full-QCD results (diamonds) are consistent with the old quenched results (circles) which are also shown: no mass dependence is seen. The weighted average of the three fullQCD points is $g_{A} / g_{V}=1.21(8)$ and is consistent 


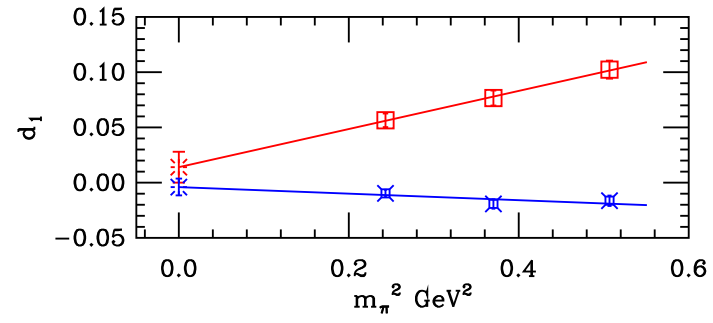

Figure 4. The $u$ (square) and $d$ quark (cross) contributions to the $d_{1}$ matrix element in full QCD.

with the quenched results.

In Fig. 2 we present the quark momentum fraction in quenched (circle) and full QCD (diamond). Though not yet renormalized, in the quenched approximation it does not show any curvature toward the chiral limit. The first moment of the transversity is shown in Fig. 3 In both the quenched and full QCD we obtain the chiral limit of the bare matrix element by linear extrapolation. We use the RI-MOM scheme with one loop running to non-perturbatively renormalize the matrix element. The quenched result is $1.194(25)(\overline{\mathrm{MS}}, 2 \mathrm{GeV})$ and the full QCD result will be reported soon.

We have also calculated the $d_{1}$ matrix element for which lack of chiral symmetry results in a mixing with a lower dimensional operator. We reported last year that this mixing is suppressed with domain wall fermions in the quenched approximation [8]. Our full-QCD $d_{1}$ results, shown in Fig. [4 are again in sharp contrast with the unsubtracted Wilson fermion results indicating that DWF suppress the power divergent mixing.

To summarize, the quenched calculations with DBW2 and DWF works well for nucleon matrix elements. The axial to vector charge ratio, $g_{A} / g_{V}$, is well reproduced to within $5 \%$ of the experimental value even on a modest volume and relatively heavy quark mass. The dependence on quark mass is very mild. Likewise the moments of structure functions $\langle x\rangle_{q}, d_{1}$, and $\langle 1\rangle_{\delta q}$ all show mild linear dependence on quark mass, though some of them are yet to be renormalized. The full-QCD calculations of these quantities seem to work as well. Similar mild linear dependence on the quark mass was observed, extrapolating to es- timates not contradicting with the quenched ones (where renormalization is done), albeit with limited statistics. With QCDOC computer [10, we will be adding more observables such as flavorsinglet quantities and form factors and higher moments of structure functions that require finite momentum, and moving to larger lattice volumes and smaller quark masses.

\section{REFERENCES}

1. D.B. Kaplan, Phys. Lett. B 288, 342 (1992); Y. Shamir, Nucl. Phys. B 406, 90 (1993); R. Narayanan and H. Neuberger, Phys. Lett. B 302, 62 (1993); V. Furman and Y. Shamir, Nucl. Phys. B 439, 54 (1995).

2. T. Blum et al. [RBC], hep-lat/0007038 to appear in Phys. Rev. D; Phys. Rev. D 66, 014504 (2002); Phys. Rev. D 65, 014504 (2002); RBRC Scientific Articles 4; hep-lat/0110075, to appear in Phys. Rev. D.

3. S. Sasaki et al., Phys. Rev. D65, 074503 (2002).

4. T. Takaishi, Phys. Rev. D 54, 1050 (1996); P. de Forcrand et al. [QCD-TARO], Nucl. Phys. B 577, 263 (2000);Y. Aoki et al., hep-lat/0211023

5. M. Fukugita et al., Phys. Rev. Lett. 75, 2092 (1995); K. F. Liu et al., Phys. Rev. D 49, 4755 (1994); P. Rakow et al., Phys. Rev. D 53, 2317 (1996); S. Güsken et al. [TXL], Phys. Rev. D 59, 114502 (1999); D. Dolgov et al. [LHPC], Phys. Rev. D 66, 034506 (2002), hep-lat/0201021 S. Capitani et al., Nucl. Phys. Proc. Suppl. 79, 548 (1999); R. Horsley [UKQCD], Nucl. Phys. Proc. Suppl. 94, 307 (2001).

6. S. Sasaki et al., hep-lat/0306007, to appear in Phys. Rev. D.

7. K. Hagiwara et al. [Particle Data Group], Phys. Rev. D 66, 010001 (2002).

8. K. Orginos [RBC], in Proc. "Lattice 2002," Nucl. Phys. B Proc. Suppl. 119, 386 (2003); in Proc. "Spin 2002," hep-lat/0211025

9. C. Dawson, in these proceedings.

10. T. Wettig and K. Petrov in these proceedings. 